\title{
Polarization dependence of EXAFS and XANES spectra of superconducting films on the basis of $\mathrm{Y}-\mathrm{Ba}-\mathrm{Cu}-\mathrm{O}$
}

\author{
N V BAUSK, L N MAZALOV, A I RYKOV, V F VRATSKIKH, \\ V R PREDTETCHENSKII and YU D VARLAMOV \\ Institute of Inorganic Chemistry, Siberian Branch of the Academy of Sciences of the USSR, \\ 630090 Novosibirsk, Prospekt Lavrentyeva 3, Novosibirsk, USSR

\begin{abstract}
Superconducting films of $\mathrm{Y}-\mathrm{Ba}-\mathrm{Cu}-\mathrm{O}$ have been studied by EXAFS and XANES spectroscopy. Films of thickness $1000 \AA$ obtained by laser sputtering onto sapphire substrates had $T_{\mathrm{c}}$ of about $90 \mathrm{~K}, \Delta T_{\mathrm{c}}$ of about $2 \mathrm{~K}$ and the $\bar{c}$ axis orientation normal to the substrate surface. The EXAFS and XANES spectra were measured in the region of $Y$ and $\mathrm{CuK}$ edges at different orientations of the sample with respect to the polarization vector $\bar{E}$. The spectra were measured in the surface-sensitive total electron yield detection mode. The observed differences between the spectra are discussed and compared with theoretical calculations.
\end{abstract}

Keywords. Polarization dependence; EXAFS; XANES; Y-Ba-Cu-O

\section{Introduction}

XANES and EXAFS spectroscopy are widely used in studies of new ceramic materials exhibiting high $T_{\mathrm{c}}$ superconductivity (Maeda et al 1987; Gygax et al 1987; Zhang et al 1988; Alp et al 1987; Antonioni et al 1987; Imazumi et al 1988). XANES can provide information about the charge state of atoms, while EXAFS enables us to determine not only the interatomic distances and coordination numbers but also the temperature dependence of the Debye-Waller factor for different pairs of atoms (Maeda et al 1987; Gygax et al 1987; Zhang et al 1988). Analysing the XANES data involves certain difficulties which may sometimes result in obtaining quite opposite conclusions from the same set of data. For example CuK-XANES has been interpreted to indicate the presence of $\mathrm{Cu}(+3)$ by Alp et al (1987) and Antonioni et al (1987) and a mixture of $\mathrm{Cu}(2+)$ and $\mathrm{Cu}(1+)$ by Imazumi et al (1988). The analysis of EXAFS data also involves some ambiguity. Thus, it has been shown that the $\mathrm{Cu}-\mathrm{O}$ atomic pairs in the $\bar{a} \bar{b}$ plane in the $\mathrm{YBa}_{2} \mathrm{Cu}_{3} \mathrm{O}_{7-\delta}$ ceramic give a weak temperature dependence of the Debye-Waller factor (i.e. this bond is the most rigid in the system) without any anomalies (Zhang et al 1988). At the same time, for the $\mathrm{Cu}-\mathrm{O}$ pairs whose oxygen atoms lie between the $\mathrm{CuO}_{2}$ "layers" and $\mathrm{CuO}$ "chains" there was an anomalous increase of the Debye-Waller factor as the temperature was decreased below $T_{c}$ (Maeda et al 1987). An anomalous increase of the Debye-Waller factor for the same atomic pairs has also been observed by Gygax et al (1987) at $170^{\circ} \mathrm{K}$. On the basis of these two latter observations the role of the "soft mode" in the high $T_{\mathfrak{c}}$ superconductivity mechanism is discussed in these studies. The complications in the analysis of the temperature behaviour of the Debye-Waller factor arise due to the presence in the $\mathrm{YBa}_{2} \mathrm{Cu}_{3} \mathrm{O}_{7-\delta}$ system of several close $\mathrm{Cu}-\mathrm{O}$ distances which are not resolved in the atomic radial distribution function. To determine the Debye-Waller factors for different $\mathrm{Cu}-\mathrm{O}$ distances fitting procedures with a large number of fitting parameters are used which introduces a degree of ambiguity. Some of the problems in the analysis of the temperature dependence of the $\mathrm{D}-\mathrm{W}$ factor in the $\mathrm{Y}-\mathrm{Ba}-\mathrm{Cu}-\mathrm{O}$ system can be 
avoided by employing the polarization dependence of EXAFS. There are two types of copper atoms present in $\mathrm{YBa}_{2} \mathrm{Cu}_{3} \mathrm{O}_{7-\delta}$-in the $\mathrm{CuO}$ "layers" and $\mathrm{CuO}$ "chains". The copper atoms in the chains have an environment close to the planar-square one (at large oxygen content) and those in the "layers" have a pyramidal oxygen environment which can be considered as planar-square since the fifth top oxygen atom lies far away from the copper atom. A more informative polarization variant of CuK-XANES can be analysed on the basis of the available experimental (Kozugi et al 1984) and theoretical (Garg et al 1988; Kozugi et al 1984) data.

\section{Experiment}

Realization of the polarization methods requires monocrystalline objects of sufficiently large dimensions. The available single crystals were unstable even during a short storage time, forming various phases on their surfaces. The studies were performed on $1000 \AA$ thick films of the composition $\mathrm{YBa}_{2} \mathrm{Cu}_{3} \mathrm{O}_{7-\delta}$ prepared by laser-sputtering onto sapphire substrates (Varlamov et al 1988). The film parameters were as follows: $F_{c}=90 \mathrm{~K}$, the transition width $\Delta T_{c}=2^{\circ} \mathrm{K}$, the critical current density was about $10^{6}$ $\mathrm{A} / \mathrm{cm}^{2}$ at $78 \mathrm{~K}$. According to the X-ray diffraction data, the $\bar{C}$ axis in such a film is oriented perpendicular to the substrate surface with no ordering in the $\bar{A}$ and $\bar{B}$ directions.

In the case of films, the use of transmission mode is impossible due to the presence of a massive substrate. The use of fluorescence method for such objects is also not optimal since the depth of penetration into a sample of X-rays with an energy of $8 \mathrm{keV}$ is considerably greater than $1000 \AA$. To study the present film by EXAFS and XANES methods we made use of the most acceptable procedure based on total electron yield detection (Stohr et al 1979).

\section{Discussion}

Figure 1 shows CuK-XANES spectra of a high $T_{c}$ superconducting film of the composition $\mathrm{YBa}_{2} \mathrm{Cu}_{3} \mathrm{O}_{7-\delta}$ obtained in total photoyield detection mode at different orientations of the polarization vector $\bar{E}$ with respect to axis $\bar{C}$. The same figure also shows a derivative of the absorption coefficient displaying more clearly the spectral features. The feature $C$ following the main maximum of the spectrum has been ascribed by some authors to the presence in $\mathrm{YBa}_{2} \mathrm{Cu}_{3} \mathrm{O}_{7-\delta}$ of +3 copper atoms (Alp et al 1987; Antonioni et al 1987), a conclusion based on the coincidence of the energy position of this band with that of the maximum in the spectrum of $\mathrm{KCuO}_{2}$ which contains $\mathrm{Cu}(+3)$. This conclusion is supported by the absence of this feature in the first calculations for a cluster with two coordination spheres (Alp et al 1987). Garg et al calculated CuK-XANES for clusters involving 1,2,3,4 coordination spheres and only in the case of four coordination spheres was this feature reproduced. If the calculations are correct, it means that the feature under discussion is not associated with the presence of $\mathrm{Cu}(+3)$. In the same work polarization dependences of CuK-XANES have been calculated and it has been shown that feature $\mathrm{C}$ appears for $\mathrm{Cu}$ atoms in the chains at orientation $\bar{E} \perp \bar{c}$. Such dependence of feature $C$ on orientation indeed appears in the experiment (figure 1) which confirms the correctness of the calculations 


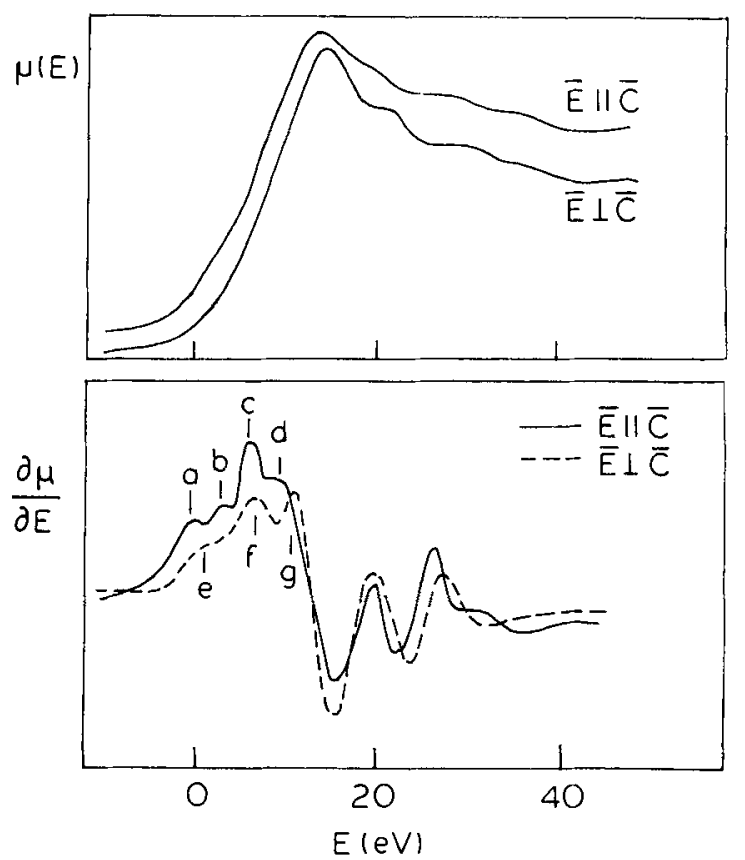

Figure 1. CuK-XANES spectra of $\mathrm{YBa}_{2} \mathrm{Cu}_{3} \mathrm{O}_{7-\delta}$ film at different orientations of the sample with respect to the polarization vector $\bar{E}$.

(Garg et al 1988). The same work also predicted a long-wave shift of the spectrum at orientation $\bar{E} \| \bar{c}$ which has been reproduced in experiment (figure 1).

A series of features at the absorption edge extending to the main maximum can be analysed on the basis of calculations in Bair and Goddard (1980) and the polarization experiment on planar-square complexes of copper (Kozugi et al 1984). It has been shown in these studies that in addition to the $1 s-4 p$ transition, that forms the main maximum, there also appear $1 s-4 p$ transitions accompanied by charge transfer from ligands to the absorbing atom (shake down satellites). The satellites are shifted by $6-8 \mathrm{eV}$ towards longer waves relative to the main transitions. The most intense satellites are observed for the $1 s-4 p_{\pi}$ transitions (i.e. for the orientation of the polarization vector perpendicular to the plane of the complex). The $1 s-4 p_{\pi}$ transition is shifted by about $5 \mathrm{eV}$ towards longer waves relative to the $1 s-4 p_{\sigma}$ transition.

Analysis of the polarization dependence of CuK-XANES in $\mathrm{YBa}_{2} \mathrm{Cu}_{3} \mathrm{O}_{7-\delta}$ is complicated by the fact that (i) the $\mathrm{CuO}_{4}$ squares in $\mathrm{CuO}_{2}$ "layers" and $\mathrm{CuO}$ "chains" are mutually perpendicular and (ii) in the films under study there are no ordering in the $A$ and $B$ directions. Nevertheless, some restrictions on the allowed transitions may be inferred. It is clear that for the $\bar{E} \| \bar{c}$ orientation there are $1 s-4 p_{\pi}$ transitions for the copper atoms in the layers and $1 s-4 p_{\sigma}$ transitions for the copper atoms in the chains (with the corresponding shakedown processes). For the orientation $\bar{E} \perp \bar{c}$ there are $1 s-4 p_{\sigma}$ transitions for the copper atoms in the "layers" and all the $1 s-4 p_{\sigma, \pi}$ transitions in the chains with an angle averaging.

The long-wave shift of the absorption edge for $\bar{E} \| \bar{c}$ can be explained by the fact that the $1 s-4 p_{\pi}$ transitions (shifted by $5 \mathrm{eV}$ towards longer waves relative to $1 s-4 p_{\sigma}$ (Kozugi et al 1984) appear in this case only for the copper atoms in the "layers". For the 
orientation $\bar{E} \perp \bar{c}$ the $1 s-4 p_{\pi}$ transitions are present only for the copper atoms in the "chains" the number of which per unit cell is one half that of the copper atoms in the "layers". In addition, the weight of the transitions due to these atoms is still more decreased, by a factor of two, due to the averaging over the angle.

In the $\bar{E} \perp \bar{c}$ spectrum the most long-wave structure (features a,b,e) must be depressed because of the forbiddenness of the $1 s-4 p_{\pi}+$ shakedown process for the copper atoms in the layers which is allowed only for copper atoms in the "chains" and is depressed by the averaging over the angle. Such a picture is observed in the experimental spectrum (figure 1): the feature e at $\bar{E} \perp \bar{c}$ is shifted towards longer waves as compared with feature $a$ for $\bar{E} \| \bar{c}$. The spectral features at shorter waves (c, d) for $\bar{E} \| \bar{c}$ are $1 s-4 p_{\pi}$ transitions for copper atoms in the "layers" and $1 s-4 p_{a}$ for copper atoms in the "chains", respectively.

In the $E \perp c$ spectra there appears feature e which is $1 s-4 p_{\pi}$ transition + shakedown for copper atoms in "chains", feature $f$ which is $1 s-4 p_{\pi}$ transition for copper atoms in "chains" and $1 s-4 p_{\pi}$ for copper atoms in "layers", $g$ is $1 s-4 p_{\sigma}$ transition for copper atoms in "chains". Thus, in the spectra there appear features a,b,e, which are shakedown satellites, $\mathrm{c}, \mathrm{f}$, which are $1 s-4 p_{\pi}$ transitions and $\mathrm{d}, \mathrm{g}$ which are $1 s-4 p_{\sigma}$ transitions.

For obtaining a clearer picture it is necessary to perform experiments on films of the composition $\mathrm{YBa}_{2} \mathrm{Cu}_{3} \mathrm{O}_{7-\delta}$ in which the "chains" are destroyed and the atoms become linearly coordinated with respect to oxygen. In this case at different orientations there will appear $1 s-4 p_{\sigma}$ or $1 s-4 p_{\pi}$ transitions without mixing.

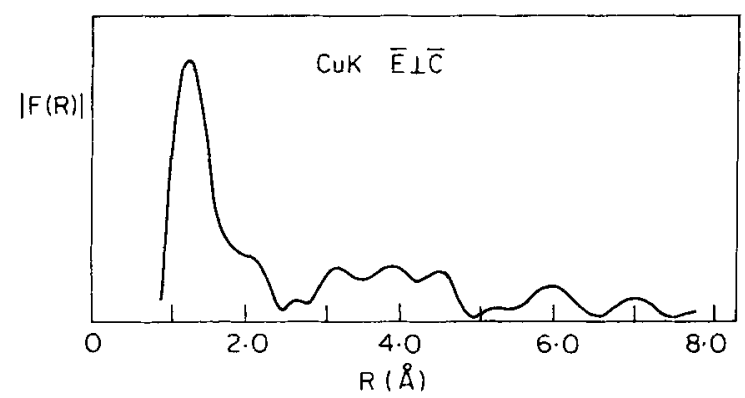

Figure 2. Atomic radial distribution function obtained by processing CuK-EXAFS spectrum of a $\mathrm{YBa}_{2} \mathrm{Cu}_{3} \mathrm{O}_{7-s}$ film at the $\bar{E} \perp \bar{c}$ orientation of the sample with respect to the polarization vector $\bar{E}$.

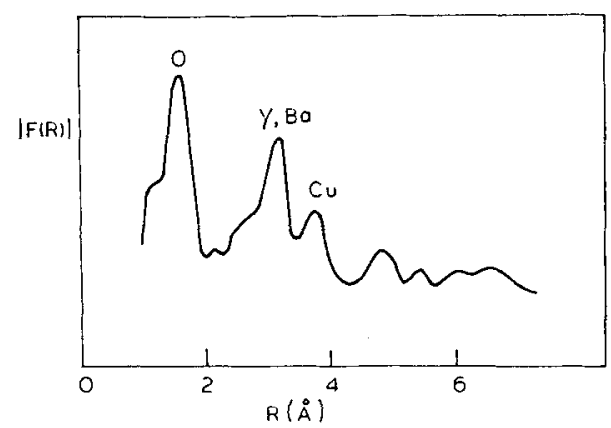

Figure 3. Atomic radial distribution function obtained by processing CuK-EXAFS spectra of polycrystalline $\mathrm{YBa}_{2} \mathrm{Cu}_{3} \mathrm{O}_{7-s}$ sample. 
It was possible to accumulate a statistics in the region of $\mathrm{CuK}$-absorption edge for $\mathrm{YBa}_{2} \mathrm{Cu}_{3} \mathrm{O}_{7-\delta}$ high $T_{c}$ superconducting film for $\bar{E} \perp \bar{c}$ which was sufficiently large to be processed. The atomic radial distribution function obtained in the processing of this spectrum is shown in figure 2 . For comparison, in figure 3 is given the radial function for a polycrystalline sample. It can be seen from comparison that, as expected, the contributions from the coordination spheres of $\mathrm{Y}$ and $\mathrm{Ba}$ atoms are supressed and there is no contribution from the oxygen atoms connecting the "layers" with "chains". Similar changes in the atomic radial distribution function have been obtained for $\bar{E} \| \bar{c}$ where the contributions from the $\mathrm{Y}, \mathrm{Ba}, \mathrm{Cu}$ atoms are depressed and there appear only oxygen atoms connecting the layers and the chains.

\section{Conclusion}

It has been shown that polarization variants of EXAFS and XANES allow us to obtain additional information on high $T_{c}$ materials. In the study of a monocrystalline film of $\mathrm{YBa}_{2} \mathrm{Cu}_{3} \mathrm{O}_{7-\delta}$ the dependence on the orientation of the sample with respect to the polarization vector has been established for feature $\mathrm{C}$ in $\mathrm{CuK}-\mathrm{XANES}$ spectrum which confirms the calculations and refutes the presence of $\mathrm{Cu}(+3)$. The observed shift of the absorption edge at $\bar{E} \| \bar{c}$ is in agreement with the existing ideas about the nature of the polarization dependence of XANES in the square-planar-square complexes of copper. The study of polarization dependence enables us to ascribe a series of features in XANES spectra to the specific transitions and shakedown satellites.

\section{Acknowledgements}

This study was performed using the synchrotron radiation of the VEPP-3 storage ring of the Institute of Nuclear Physics of the Siberian Branch of the USSR Academy of Sciences, Novosibirsk.

\section{References}

Alp E E et al 1987 Phys. Rev. B35 7199

Antonioni G M et al 1987 Eur. Lett. 4851

Bair R A and Goddard III W A 1980 Phys. Rev. B22 2767

Garg K B et al 1988 Phys. Rev. B38 244

Gygax S et al 1987 Jap. J. Appl. Phys. Suppl. 261051

Imazumi T et al 1988 Solid State Commun. 65213

Kozugi N et al 1984 Chem. Phys. 91249

Maeda H et al 1987 Phys. Soc. Jap. 563413

Stohr J et al 1979 Phys. Rev. B20 664

Yu D Varlamov, Vratskikh V F and Predtetchenskii M R 1988 Preprint No. 190, Siberian Branch of the USSR Acad. Sci. Novosibirsk pp 12 (in Russian) (Novosibirsk: Institute of Thermal Physics)

Zhang K et al 1988 Phys. Rev. B37 3375 